\title{
Remarks on strongly convex stochastic processes
}

\author{
DAWID KOTRYS
}

\begin{abstract}
Strongly convex stochastic processes are introduced. Some well-known results concerning convex functions, like the Hermite-Hadamard inequality, Jensen inequality, Kuhn theorem and Bernstein-Doetsch theorem are extended to strongly convex stochastic processes.
\end{abstract}

Mathematics Subject Classification (1991). Primary 26A51; Secondary 26D15, 39B62, 60 G99.

Keywords. Strongly convex stochastic process, Hermite-Hadamard inequality,

Jensen inequality, Bernstein-Doetsch theorem, Kuhn theorem, mean-square integral.

\section{Introduction}

In 1980 Nikodem [7] considered convex stochastic processes. In 1995 Skowroński [9] obtained some further results on convex stochastic processes, which generalize some known properties of convex functions. Moreover, in a recent paper [3], the present author showed the Hermite-Hadamard-type inequality for convex stochastic processes.

Let $I \subset \mathbb{R}$ be an interval. Recall that a function $f: I \rightarrow \mathbb{R}$ is called strongly convex with modulus $c>0$, if

$$
f(\lambda x+(1-\lambda) y) \leqslant \lambda f(x)+(1-\lambda) f(y)-c \lambda(1-\lambda)(x-y)^{2}
$$

for any $x, y \in I$ and $\lambda \in[0,1]$ (cf. [2,8]). Obviously, every strongly convex function is convex. Observe also that, for instance, affine functions are not strongly convex.

In this paper we propose the generalization of convexity of this kind for stochastic processes. Let $(\Omega, \mathcal{A}, P)$ be an arbitrary probability space. A function $X: \Omega \rightarrow \mathbb{R}$ is called a random variable, if it is $\mathcal{A}$-measurable. A function $X: I \times \Omega \rightarrow \mathbb{R}$ is called a stochastic process, if for every $t \in I$ the function $X(t, \cdot)$ is a random variable. 
Let $C: \Omega \rightarrow \mathbb{R}$ denote a positive random variable. We say that a stochastic process $X: I \times \Omega \rightarrow \mathbb{R}$ is strongly convex with modulus $C(\cdot)>0$, if the inequality

$$
X(\lambda u+(1-\lambda) v, \cdot) \leqslant \lambda X(u, \cdot)+(1-\lambda) X(v, \cdot)-C(\cdot) \lambda(1-\lambda)(u-v)^{2} \quad \text { (a.e.) }
$$

is satisfied for all $\lambda \in[0,1]$ and $u, v \in I$. If the above inequality is assumed only for $\lambda=\frac{1}{2}$, then the process $X$ is strongly Jensen-convex with modulus $C(\cdot)$ or strongly midconvex with modulus $C(\cdot)$. If the above inequality holds for a fixed number $\lambda \in(0,1)$, then we say that the process $X$ is strongly $\lambda$-convex with modulus $C(\cdot)$.

Obviously, by omitting the term $C(\cdot) \lambda(1-\lambda)(u-v)^{2}$ in inequality (1), we immediately get the definition of a convex stochastic process introduced by Nikodem in 1980 [7]. On the other hand, we derive it from (1) in a limit case, when $C(\cdot) \equiv 0$.

The main subject of this paper is to extend some well-known results concerning convex functions to strongly convex stochastic processes. We obtain the counterparts of the Hermite-Hadamard inequality, Jensen inequality, Kuhn theorem and Bernstein-Doetsch theorem. In the deterministic case most of the presented results reduce to the properties of strongly convex functions obtained recently in [1] and [6]. Note also that the related results for convex stochastic processes can be found in $[3,7,9]$.

\section{Jensen-type inequality}

In this section we present a Jensen-type inequality for strongly convex stochastic processes. In [3] the following proposition was shown.

Proposition 1. Let $X: I \times \Omega \rightarrow \mathbb{R}$ be a convex stochastic process and $t_{0} \in \operatorname{int} I$. Then there exists a random variable $A: \Omega \rightarrow \mathbb{R}$ such that $X$ is supported at $t_{0}$ by the process $A(\cdot)\left(t-t_{0}\right)+X\left(t_{0}, \cdot\right)$. That is

$$
X(t, \cdot) \geqslant A(\cdot)\left(t-t_{0}\right)+X\left(t_{0}, \cdot\right) \quad \text { (a.e.) }
$$

for all $t \in I$.

We begin our investigations with an easy but very useful lemma. Of course, its version for strongly convex functions is well-known (cf. e.g. [2]).

Lemma 2. A stochastic process $X: I \times \Omega \rightarrow \mathbb{R}$ is strongly $\lambda$-convex (strongly convex, respectively) with modulus $C(\cdot)$ if and only if the stochastic process $Y: I \times \Omega \rightarrow \mathbb{R}$ defined by $Y(t, \cdot):=X(t, \cdot)-C(\cdot) t^{2}$ is $\lambda$-convex (convex, respectively). 
Proof. In the first part of the proof assume that $X$ is strongly $\lambda$-convex. Fix $u, v \in I$. By strong $\lambda$-convexity we get

$$
\begin{aligned}
Y(\lambda u+(1-\lambda) v, \cdot)= & X(\lambda u+(1-\lambda) v, \cdot)-C(\cdot)(\lambda u+(1-\lambda) v)^{2} \\
\leqslant & \lambda X(u, \cdot)+(1-\lambda) X(v, \cdot) \\
& -C(\cdot)\left(\lambda(1-\lambda)(u-v)^{2}+(\lambda u+(1-\lambda) v)^{2}\right) \\
= & \lambda X(u, \cdot)+(1-\lambda) X(v, \cdot)-C(\cdot)\left(\lambda u^{2}+(1-\lambda) v^{2}\right) \\
= & \lambda\left(X(u, \cdot)-C(\cdot) u^{2}\right)+(1-\lambda)\left(X(v, \cdot)-C(\cdot) v^{2}\right) \\
= & \lambda Y(u, \cdot)+(1-\lambda) Y(v, \cdot) \quad \text { (a.e.). }
\end{aligned}
$$

The proof of the second part is similar, so we omit it.

If $\lambda \in[0,1]$ is arbitrarily chosen, then we obtain the lemma for strongly convex stochastic processes.

From Lemma 2 and Proposition 1 we immediately derive

Corollary 3. If a stochastic process $X: I \times \Omega \rightarrow \mathbb{R}$ is strongly convex with modulus $C(\cdot)$, then for all $t_{0} \in \operatorname{int} I, X$ is supported at $t_{0}$ by the process $H$ : $I \times \Omega \rightarrow \mathbb{R}$ of the form

$$
H(t, \cdot)=C(\cdot)\left(t-t_{0}\right)^{2}+A(\cdot)\left(t-t_{0}\right)+X\left(t_{0}, \cdot\right) .
$$

Now we present a Jensen-type theorem for strongly convex stochastic processes.

Theorem 4. Let $X: I \times \Omega \rightarrow \mathbb{R}$ be a strongly convex stochastic process with modulus $C(\cdot)$. Then

$$
X\left(\sum_{i=1}^{n} \lambda_{i} t_{i}, \cdot\right) \leqslant \sum_{i=1}^{n} \lambda_{i} X\left(t_{i}, \cdot\right)-C(\cdot) \sum_{i=1}^{n} \lambda_{i}\left(t_{i}-\bar{t}\right)^{2} \quad \text { (a.e.) }
$$

for all $t_{1}, \ldots, t_{n} \in I, \lambda_{1}, \ldots, \lambda_{n}>0$, such that $\lambda_{1}+\cdots+\lambda_{n}=1$ and $\bar{t}=$ $\lambda_{1} t_{1}+\cdots+\lambda_{n} t_{n}$.

Proof. Let us take $t_{1}, \ldots, t_{n} \in I$ and $\lambda_{1}, \ldots, \lambda_{n}>0$, such that $\lambda_{1}+\cdots+\lambda_{n}=1$. We put $\bar{t}=\lambda_{1} t_{1}+\cdots+\lambda_{n} t_{n}$. According to Corollary 3 , we have the support

$$
H(t, \cdot)=C(\cdot)(t-\bar{t})^{2}+A(\cdot)(t-\bar{t})+X(\bar{t}, \cdot)
$$

at $\bar{t}$. Then for each $i \in\{1, \ldots, n\}$ we have

$$
\left.X\left(t_{i}, \cdot\right) \geqslant H\left(t_{i}, \cdot\right)=C(\cdot)\left(t_{i}-\bar{t}\right)^{2}+A(\cdot)\left(t_{i}-\bar{t}\right)+X(\bar{t}, \cdot) \quad \text { (a.e. }\right) .
$$

Multiplying the above inequality by $\lambda_{i}$ and summing up all the inequalities we have

$$
\left.\sum_{i=1}^{n} \lambda_{i} X\left(t_{i}, \cdot\right) \geqslant C(\cdot) \sum_{i=1}^{n} \lambda_{i}\left(t_{i}-\bar{t}\right)^{2}+A(\cdot) \sum_{i=1}^{n} \lambda_{i}\left(t_{i}-\bar{t}\right)+X(\bar{t}, \cdot) \quad \text { (a.e. }\right)
$$


Because $\sum_{i=1}^{n} \lambda_{i}\left(t_{i}-\bar{t}\right)=0$,

$$
\left.X\left(\sum_{i=1}^{n} \lambda_{i} t_{i}, \cdot\right) \leqslant \sum_{i=1}^{n} \lambda_{i} X\left(t_{i}, \cdot\right)-C(\cdot) \sum_{i=1}^{n} \lambda_{i}\left(t_{i}-\bar{t}\right)^{2} \quad \text { (a.e. }\right) .
$$

\section{Kuhn-type and Bernstein-Doetsch-type results}

The classical result due to Kuhn (cf. [5]) states that if $f: I \rightarrow \mathbb{R}$ fulfils, for some fixed $\lambda \in(0,1)$ and for all $x, y \in I$, the inequality

$$
f(\lambda x+(1-\lambda) y) \leqslant \lambda f(x)+(1-\lambda) f(y),
$$

i.e. $f$ is a $\lambda$-convex function, then $f$ is also Jensen-convex, which means that

$$
f\left(\frac{x+y}{2}\right) \leqslant \frac{f(x)+f(y)}{2}, \quad x, y \in I .
$$

Skowroński proved in [9] that a $\lambda$-convex stochastic process is also Jensenconvex. In this section we prove the counterparts of these facts for strongly $\lambda$-convex stochastic processes.

Theorem 5. Let $\lambda \in(0,1)$ be a fixed number and $X: I \times \Omega \rightarrow \mathbb{R}$ be a strongly $\lambda$-convex stochastic process with modulus $C(\cdot)$. Then $X$ is Jensen-convex with modulus $C(\cdot)$.

Proof. Assume that $X$ is strongly $\lambda$-convex. Lemma 2 yields that the process $Y(t, \cdot)=X(t, \cdot)-C(\cdot) t^{2}$ is $\lambda$-convex. By Skowroński's result $Y$ is midconvex, which means that

$$
Y\left(\frac{u+v}{2}, \cdot\right) \leqslant \frac{Y(u, \cdot)+Y(v, \cdot)}{2} \quad \text { (a.e.) }
$$

Therefore

$X\left(\frac{u+v}{2}, \cdot\right)-C(\cdot)\left(\frac{u+v}{2}\right)^{2} \leqslant \frac{X(u, \cdot)-C(\cdot) u^{2}+X(v, \cdot)-C(\cdot) v^{2}}{2} \quad$ (a.e.)

and after some rearrangement we arrive at

$$
X\left(\frac{u+v}{2}, \cdot\right) \leqslant \frac{X(u, \cdot)+X(v, \cdot)}{2}-\frac{C(\cdot)}{4}(u-v)^{2} \quad \text { (a.e.) },
$$

which finishes the proof.

It is well-known that a midconvex function $f: I \rightarrow \mathbb{R}$ is convex under slight regularity assumptions, like local upper boundedness at some point (BernsteinDoetsch Theorem) or measurability (Sierpiński's Theorem), also under some other assumptions of this type (cf. [4]).

Nikodem presented in [7] the conditions guaranteeing the convexity of midconvex stochastic processes. Now we consider a similar problem for strongly 
convex processes. We would like to recall the following definitions. A stochastic process $X: I \times \Omega \rightarrow \mathbb{R}$ is called

(i) P-upper bounded on the interval $(a, b) \subset I$, iff

$$
\lim _{n \rightarrow \infty} \sup _{t \in(a, b)}\{P(\{\omega \in \Omega: X(t, \omega) \geqslant n\})\}=0,
$$

(ii) continuous in probability in interval $I$, if for all $t_{0} \in I$ we have

$$
P-\lim _{t \rightarrow t_{0}} X(t, \cdot)=X\left(t_{0}, \cdot\right),
$$

where $P-\lim$ denotes the limit in probability.

For more details we refer the reader to [7]. Now we shall prove the following

Theorem 6. If a stochastic process $X: I \times \Omega \rightarrow \mathbb{R}$ is strongly midconvex with modulus $C(\cdot)$ and $P$-upper bounded on the interval $(a, b) \subset I$, then it is continuous in the interval $I$.

Proof. Being strongly a midconvex, $X$ is also a midconvex stochastic process. Since $X$ is $\mathrm{P}$-upper bounded on the interval $(a, b)$, it is continuous in view of [7, Theorem 4].

Theorem 7. Assume that $I$ is an open interval. A strongly midconvex stochastic process $X: I \times \Omega \rightarrow \mathbb{R}$ with modulus $C(\cdot)$ is continuous if and only if it is strongly convex with modulus $C(\cdot)$.

Proof. To prove necessity take the process $Y(t, \cdot)=X(t, \cdot)-C(\cdot) t^{2}$. By Lemma 2 we get that $Y$ is midconvex. Since $X$ is continuous, $Y$ is also continuous. Using Nikodem's result [7, Theorem 5] we arrive at that $Y$ is convex. Using Lemma 2 once more, we infer that $X$ is strongly convex with modulus $C(\cdot)$.

To prove sufficiency we observe that if $X$ is strongly convex, then $X$ is also convex. By Nikodem's result [7, Theorem 5] we get its continuity.

By Theorems 5 and 7 we obtain immediately

Corollary 8. If a process $X: I \times \Omega \rightarrow \mathbb{R}$ is continuous and strongly $\lambda$-convex with modulus $C(\cdot)$, then it is strongly convex with modulus $C(\cdot)$.

\section{Hermite-Hadamard-type inequality}

It is well-known that every convex function $f: I \rightarrow \mathbb{R}$ satisfies the HermiteHadamard inequality

$$
f\left(\frac{x+y}{2}\right) \leqslant \frac{1}{y-x} \int_{x}^{y} f(s) d s \leqslant \frac{f(x)+f(y)}{2} .
$$


for any $x, y \in I$. This celebrated result plays a very important role in convex analysis. In [3, Theorem 3] its counterpart for convex stochastic processes was presented. Below we quote this result. Let us recall before that a stochastic process $X: I \times \Omega \rightarrow \mathbb{R}$ is mean-square continuous in the interval $I$, if for all $t_{0} \in I$ the condition $\lim _{t \rightarrow t_{0}} E\left(\left|X(t)-X\left(t_{0}\right)\right|^{2}\right)=0$ holds.

Theorem 9. If $X: I \times \Omega \rightarrow \mathbb{R}$ is a Jensen-convex, mean-square continuous stochastic process in the interval $I$, then for any $u, v \in I$ we have

$$
X\left(\frac{u+v}{2}, \cdot\right) \leqslant \frac{1}{v-u} \int_{u}^{v} X(t, \cdot) \mathrm{d} t \leqslant \frac{X(u, \cdot)+X(v, \cdot)}{2} \quad \text { (a.e.). }
$$

The integral in the statement is mean-square integral. For the definition and basic properties of mean-square integral see for example [10]. Now we would like to prove the Hermite-Hadamard inequality for strongly convex stochastic processes. We start with a technical lemma.

Lemma 10. Let $X: I \times \Omega \rightarrow \mathbb{R}$ be the stochastic process of the form $X(t, \cdot)=$ $C(\cdot) t^{2}$, where $C: \Omega \rightarrow \mathbb{R}$ is a random variable, such that $E\left[C^{2}\right]<\infty$. If $[u, v] \subset I$, then

$$
\int_{u}^{v} X(t, \cdot) \mathrm{d} t=C(\cdot) \frac{v^{3}-u^{3}}{3}
$$

Proof. By elementary properties of the expectation we have

$$
\begin{aligned}
E\left[\sum_{i=1}^{n} X\left(\Theta_{i}\right)\left(t_{i}-t_{i-1}\right)-C \frac{v^{3}-u^{3}}{3}\right]^{2} & =E\left[\sum_{i=1}^{n} C \Theta_{i}^{2}\left(t_{i}-t_{i-1}\right)-C \frac{v^{3}-u^{3}}{3}\right]^{2} \\
& =E\left[C\left(\sum_{i=1}^{n} \Theta_{i}^{2}\left(t_{i}-t_{i-1}\right)-\frac{v^{3}-u^{3}}{3}\right)\right]^{2} \\
& =\left(\sum_{i=1}^{n} \Theta_{i}^{2}\left(t_{i}-t_{i-1}\right)-\frac{v^{3}-u^{3}}{3}\right)^{2} E\left[C^{2}\right] .
\end{aligned}
$$

If $n \rightarrow \infty$, then the above expression tends to zero, because of the definition of the Riemann integral. This finishes the proof.

Theorem 11. Let $X: I \times \Omega \rightarrow \mathbb{R}$ be a stochastic process, which is strongly Jensen-convex with modulus $C(\cdot)$ and mean-square continuous in the interval I. Then for any $u, v \in I$ we have 


$$
\begin{aligned}
& X\left(\frac{u+v}{2}, \cdot\right)+C(\cdot) \frac{(v-u)^{2}}{12} \\
& \leqslant \frac{1}{v-u} \int_{u}^{v} X(t, \cdot) \mathrm{d} t \leqslant \frac{X(u, \cdot)+X(v, \cdot)}{2}-C(\cdot) \frac{(u-v)^{2}}{6} \quad \text { (a.e.). }
\end{aligned}
$$

Proof. According to the assumption the process $X$ is strongly convex with modulus $C(\cdot)$, so by Lemma 2 the process $Y(t, \cdot)=X(t, \cdot)-C(\cdot) t^{2}$ is convex. By inequality (2) we get

$$
\begin{aligned}
Y\left(\frac{u+v}{2}, \cdot\right) & \leqslant \frac{1}{v-u} \int_{u}^{v} Y(t, \cdot) \mathrm{d} t \\
& \leqslant \frac{Y(u, \cdot)+Y(v, \cdot)}{2} \quad \text { (a.e.). }
\end{aligned}
$$

Hence

$$
\begin{aligned}
X\left(\frac{u+v}{2}, \cdot\right)-C(\cdot)\left(\frac{u+v}{2}\right)^{2} & \leqslant \frac{1}{v-u} \int_{u}^{v}\left(X(t, \cdot)-C(\cdot) t^{2}\right) \mathrm{d} t \\
& \leqslant \frac{X(u, \cdot)-C(\cdot) u^{2}+X(v, \cdot)-C(\cdot) v^{2}}{2} \quad \text { (a.e.). }
\end{aligned}
$$

Furthermore

$$
\begin{aligned}
X\left(\frac{u+v}{2}, \cdot\right)-C(\cdot)\left(\frac{u+v}{2}\right)^{2} & \leqslant \frac{1}{v-u} \int_{u}^{v} X(t, \cdot) \mathrm{d} t-\frac{1}{v-u} \int_{u}^{v} C(\cdot) t^{2} \mathrm{~d} t \\
& \left.\leqslant \frac{X(u, \cdot)+X(v, \cdot)}{2}-\frac{C(\cdot)}{2}\left(u^{2}+v^{2}\right) \quad \text { (a.e. }\right)
\end{aligned}
$$

By Lemma 10 we have

$$
\begin{aligned}
X\left(\frac{u+v}{2}, \cdot\right)-C(\cdot)\left(\frac{u+v}{2}\right)^{2} & \leqslant \frac{1}{v-u} \int_{u}^{v} X(t, \cdot) \mathrm{d} t-C(\cdot) \frac{1}{v-u} \frac{v^{3}-u^{3}}{3} \\
& \leqslant \frac{X(u, \cdot)+X(v, \cdot)}{2}-\frac{C(\cdot)}{2}\left(u^{2}+v^{2}\right) \quad \text { (a.e.). }
\end{aligned}
$$

Adding to all sides of the above inequality the term $C(\cdot) \frac{1}{v-u} \frac{v^{3}-u^{3}}{3}$ and making some simple computation, we get inequality (3).

Open Access. This article is distributed under the terms of the Creative Commons Attribution License which permits any use, distribution, and reproduction in any medium, provided the original author(s) and the source are credited. 


\section{References}

[1] Azócar, A., Giménez, J., Nikodem, K., Sánchez, J.L.: On strongly midconvex functions. Opuscula Math. 31/1, 15-26 (2011)

[2] Hiriart-Urruty, J.B., Lemaréchal, C.: Fundamentals of Convex Analysis. Springer, Berlin (2001)

[3] Kotrys, D.: Hermite-Hadamard inequality for convex stochastic processes. Aequationes Math. 83, 143-151 (2012)

[4] Kuczma, M.: An Introduction to the Theory of Functional Equations and Inequalities. Birkhäuser, Basel (2009)

[5] Kuhn, N.: A note on $t$-convex functions. In: General inequalities 4 (Oberwolfach, 1983). International Schriftenreihe Numerical Mathematics, vol. 71, pp. 269-276. Birkhäuser, Basel (1984)

[6] Merentes, N., Nikodem, K.: Remarks on strongly convex functions. Aequationes Math. 80, 193-199 (2010)

[7] Nikodem, K.: On convex stochastic processes. Aequationes Math. 20, 184-197 (1980)

[8] Roberts, A.W., Varberg, D.E.: Convex functions. Academic Press, New York, London (1973)

[9] Skowroński, A.: On Wright-convex stochastic processes. Ann. Math. Sil. 9, 29-32 (1995)

[10] Sobczyk, K.: Stochastic differential equations with applications to physics and engineering. Kluwer Academic Publishers, Dordrecht (1991)

Dawid Kotrys

Department of Mathematics and Computer Science

University of Bielsko-Biała

Willowa 2, 43-309 Bielsko-Biała, Poland

e-mail: dkotrys@ath.bielsko.pl

Received: June 1, 2012

Revised: August 28, 2012 